\title{
Spectroscopic Observations of the Mass Donor Star in SS 433
}

\section{Todd Hillwig*}

Valparaiso University, USA

E-mail: todd.hillwig@valpo.edu

\section{Doug Gies}

Georgia State University

E-mail: gies@chara.gsu.edu

We have recently detected an absorption line component in the spectrum of the microquasar SS 433. We believe this component originates from the mass donor star. The absorption line component has radial velocities opposite that of the disk, and the strength of the lines relative to the continuum varies as expected for a supergiant mass donor star in the system. The spectrum resembles that of a mid-A supergiant star. Recent observations by other groups have led to conflicting results about the nature of the absorption spectrum. Here we present results from new blue spectra of SS 433 that were made at the orbital and precessional phases when the mass donor is best seen in the foreground above the plane of the obscuring disk. The orbital phase variations of the absorption line velocities and strengths are consistent with line formation in the photosphere of an A-supergiant companion. We present new estimates of the masses of both stars: $M_{o}=12.5 \pm 3.3$ $\mathrm{M}_{\odot}$ and $M_{x}=4.4 \pm 0.8 \mathrm{M}_{\odot}$.

VI Microquasar Workshop: Microquasars and Beyond September 18-22 2006

Società del Casino, Como, Italy

\footnotetext{
* Speaker.
} 


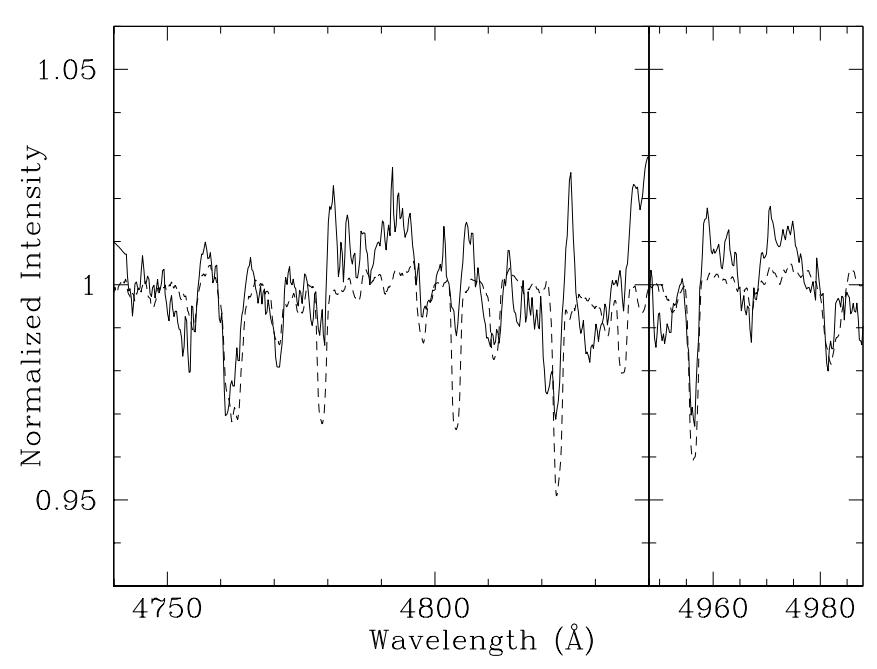

Figure 1: An overplot of the continuum region of SS 433 near mid-eclipse of the 2006 June Gemini North run (solid line) and the A4 Iab star HD 9233 (dashed line). The spectra have been Doppler shifted and the spectrum of HD 9233 intensity scaled to match the measured spectrum of SS 433.

\section{Introduction}

The claim has been made that the mass donor star in SS 433 has been observed through moderate resolution spectroscopy [1]. A mass ratio and masses for the two components were provided, though the results were considered preliminary. The identification of the mass donor was brought into question [2] with the suggestion that some of the absorption features arise in the circumstellar gas outflow. We performed new observations to clarify the source of the observed absorption spectrum.

\section{Observations}

New spectra of SS 433 were obtained in 2006 June 7-13 at the Gemini North observatory with GMOS in long-slit mode. The spectra were taken at the same system orientation as before [1], covering orbital phase, $\phi=0$, and near disk precession phase, $\psi=0$. We used the orbital ephemeris HJD 2,450,023.62+13.08211E [3], and the model precession ephemeris HJD $2,451,458.12+162.15 \mathrm{E}$ [ [ $₫$ ]. The SNR of the mid-eclipse spectrum is $\sim 200$.

\section{The Absorption Spectrum of SS 433}

For our analysis of the mass donor star, we used the spectral region 4748-4878 $\AA$ and 4950 $4985 \AA$, the best available portion continuum regions free of large emission lines. Many of the large "stationary" emission lines on each night include strong P Cygni profiles indicating a strong wind component.

Figure 1 shows the over-plotted spectra of SS 433 and the A4 Iab star HD 9233, the most closely matching spectral type. The spectra have been Doppler shifted to an identical reference 
frame for comparison. The intensity factor applied to the A star spectrum is 0.36 , as found previously [1]. The overlap shows that a mid-A supergiant spectrum still provides a good comparison to the absorption spectrum observed in SS 433.

\section{The Radial Velocity Curve of the Mass Donor Star}

To measure radial velocities, we found line centers through Gaussian fitting of the absorption features. The line centers were then compared directly to corresponding line centers for the spectrum of HD 9233, providing relative velocities. Radial velocities were obtained by adding the heliocentric radial velocity of the HD 9233 spectrum (-69.5 km s-1). Velocities for the final two nights are currently based on only one line (the strongest visible line). The HD 9233 spectrum is from a 2005 August observation with the Medium Resolution Spectrograph on the Hobby Eberly telescope. The spectrum was boxcar smoothed to match the resolution of the Gemini data.

We fit the radial velocity curve of the mass donor using both our new data and that from before [1], giving a system velocity, $\gamma=73 \pm 3 \mathrm{~km} \mathrm{~s}^{-1}$ and a semi-amplitude, $K_{o}=60 \pm 3 \mathrm{~km}$ $\mathrm{s}^{-1}$. Figure 2 shows the data, best-fit sine curve, and the $2 \sigma$ amplitude values. Using a semiamplitude for the compact object and disk of $K_{x}=168 \pm 18 \mathrm{~km} \mathrm{~s}^{-1}$ [价, we arrive at a mass ratio, $q=M_{x} / M_{o}=K_{o} / K_{x}=0.35 \pm 0.04$.

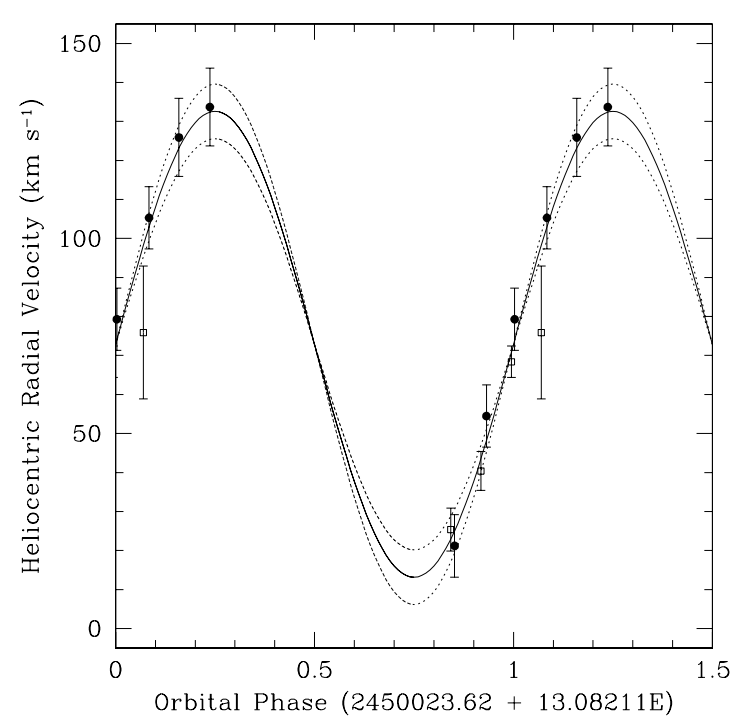

Figure 2: Prior radial velocity data [1] (hollow squares) and the new Gemini North data (filled circles) along with the model fit radial velocity curve (solid line) and $2 \sigma$ variation (dotted lines).

\section{Line Strengths in SS 433}

\subsection{Absorption Line Equivalent Width Amplitudes}

We expect that absorption lines from the mass donor will increase in strength relative to the continuum as SS 433 goes into eclipse and a larger fraction of the observed light is coming from 
the donor star. The pattern and relative strengths of the lines from the new data are consistent with previous data [1], taken 2003 October at KPNO. This strongly suggests that we are observing a stellar photosphere rather than a highly variable wind component. The steeper fall off of the data points compared to the model is consistent with our claim that at other orientations, the mass donor is hidden by opaque gas in the disk.

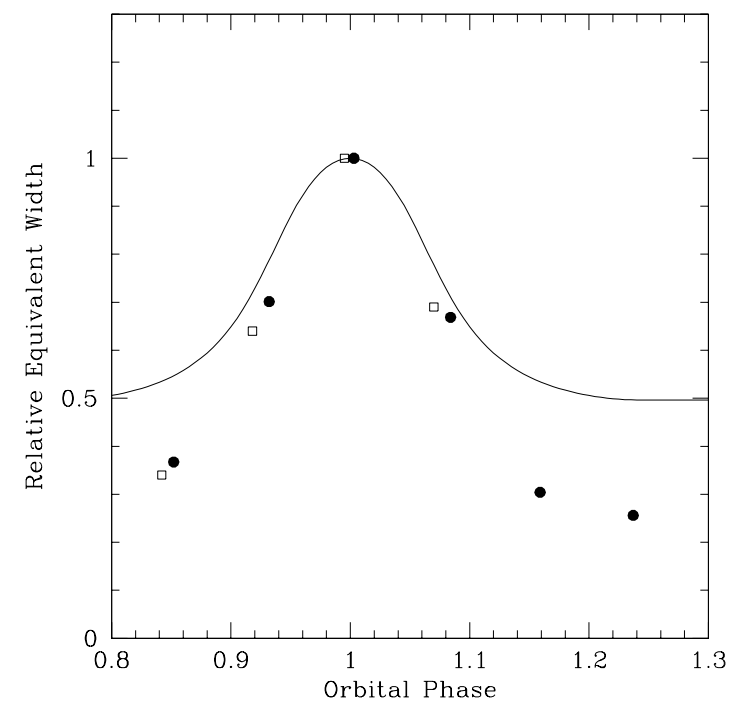

Figure 3: Relative line strengths for absorption features from the 2003 October data (hollow squares) and the 2006 June Gemini North data (filled circles). The solid line shows a model B-band light curve (inverted).

\section{2 "Stationary" Emission Lines}

Figure 4 shows the normalized relative strengths of the "stationary" emission features at $\mathrm{H} \gamma$ and He I $\lambda 4471 \AA$ for the Gemini North 2006 June spectra. Both show a clear eclipse effect centered on the night with calculated orbital phase closest to $\phi=0.0$. This confirms that our ephemeris was correct and that our spectra were taken during primary eclipse.

\section{Discussion}

\subsection{Detection of the Mass Donor}

Clearly an absorption spectrum that was not due to the mass donor star has been observed [2]. However, the observations were not obtained at what we believe to be the optimum time to see the mass donor star. Therefore we obtained new observations at the same system orientation as our previous data [1]. If the absorption spectrum were due to a wind, we would expect that the line velocities, strengths, and variability to clearly differ from our previous results. If however the absorption component we observe originates in the mass donor star, the observations should very closely match, in all respects, our previous results. Above we see that the line strength and spectrum (Figure 1), the radial velocities (Figure 2), and the relative line variability (Figure 3) all 


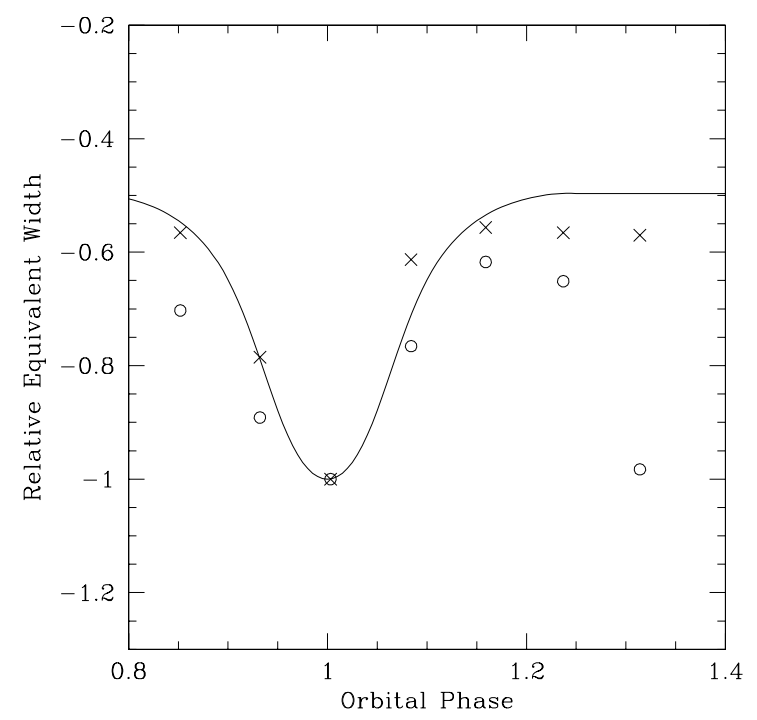

Figure 4: Equivalent width measurements for the $\mathrm{H} \gamma$ (circles) and $\mathrm{He} \mathrm{I} \lambda 4471 \AA$ (crosses) emission features plotted vs. orbital phase. The solid line shows the expected scaled B-band eclipse light curve.

match perfectly, within the errors to our previous data. We take this as strong evidence that the absorption spectrum we observe does originate in the mass donor star.

\subsection{Component Masses}

Using the $K_{o}, K_{x}$, and $P_{o r b}$ values from above, along with the accepted system inclination of $78.8^{\circ}$, we arrive at updated component masses of: $M_{o}=12.5 \pm 3.3 \mathrm{M}_{\odot}$ and $M_{x}=4.4 \pm 0.8 \mathrm{M}_{\odot}$. This places the mass donor star within in the expected mass range for A supergiant stars, and the compact object well above neutron star masses.

Financial support was provided by the National Science Foundation through grant AST-0607432 (T.C.H.)

\section{References}

[1] T.C. Hillwig, D.R. Gies, W. Huang, M.V. McSwain, M.A. Stark, A. van der Meer, \& L. Kaper, Identification of the Mass Donor Star's Spectrum in SS 433, 2004, ApJ, 615, 422

[2] A.D. Barnes, J. Casares, P.A. Charles, J.S. Clark, R. Cornelisse, C. Knigge, \& D. Steeghs, On the origin of the absorption features in SS433, 2006, MNRAS, 365, 296

[3] V.P. Goranskii, V.F. Esipov, \& A.M. Cherepashchuk, Optical variability of SS 433 in 1978-1996, 1998, Astron. Rep., 42, 209

[4] D.R. Gies, M.V. McSwain, R.L. Riddle, Z. Wang, P.J. Wiita, \& D.W. Wingert, The Spectral Components of SS 433, 2002, ApJ, 566, 1069 